\title{
Optimization of the flat stock cutting process by hydroabrasive jet
}

\author{
Mikhail A. Tamarkin ${ }^{1, *}$, Victor I. Butenko ${ }^{2}$, Albert N. Isaev ${ }^{1}$, and Elena V. Murugova ${ }^{1}$ \\ ${ }^{1}$ Don State Technical University, 344000 Rostov-on-Don, Russia \\ ${ }^{2}$ Southern Federal University, 347922 Taganrog, Russia
}

\begin{abstract}
This article focuses on the key results of hydroabrasive flat stock cutting research. The forming process is described and its theoretical investigation is presented. The theoretical dependence for the roughness determination of the surface cut is set. Experimental results of the process investigation confirming their theoretical adequacy are put forward. The next steps of process optimization within the framework of modern production are allocated.
\end{abstract}

\section{Introduction}

The hydroabrasive cutting method suitable for cutting of workpieces is distinguished among all the modern and perspective methods. A wide range of processed thickness, possibility of chemically heterogeneous material cutting (non-ferrous metals and alloys, carbonaceous and alloy steels, heat steel, titanic alloys, composites, wood, plastic, glass, stone etc.), high efficiency, production of a high quality of a surface cut, possibility of workpiece processing of complex geometry, does this method of processing the most demanded in the modern blank production. The competitive advantages of this method are also the lack of thermal impact on the material, low cutting force, the erosive nature of destruction which does not contribute to the development of internal strain in a cut zone.

A hydroabrasive material cutting is made by a thin jet of water and an abrasive mixture that is moved at a high (supersonic) speed and under high pressure. This process is an erosive destruction where the abrasive fines remove microshavings from the processed surface under the influence of a working jet. Water in this regard is the key remover of the particles from a cutting zone. The acute mineral fines of the grain size from 0.1 to $0.3 \mathrm{~mm}$. are used as an abrasive. Depending on the processed material and its thickness, the quantity of the used abrasive is from 100 to $600 \mathrm{~g} / \mathrm{min}$. The garnet sand is mainly used for the aircraft detail production, as it is the natural abrasive mineral possessing a high durability and hardness of 7.5-8.0 according to the Moose's scale and has an ideal form of grain. To guarantee a high quality of cutting the abrasive should not contain large grains and dust. Grains of the size from 80 microns to 120 microns are usually used.

Despite obvious advantages of the considered method of preforming the hydroabrasive, process of cutting is studied insufficiently. The detailed analysis of the scientific works by R.A. Tikhomirov, E.N. Petukhov, V.F. Babanin, Y.S. Stepanov, M.A. Burnashev,

\footnotetext{
*Tamarkin M.A.: tehn_rostov@mail.ru
} 
V.D. Shapiro, G.V. Barsukov, D. Arola, M. Ramulu, Y. Zhang, J Chao, J. Zeng, Shpilev V. $\mathrm{V}$. showed that there is the dependence on the dimensional characteristics of the product produced by the hydroabrasive cutting method. The influence of the cutting modes on the accuracy of a curvilinear profile of preforming is described. Opportunities of the cutting ability increase, for example, by twisting of a jet or discrete impulse of an abrasive. However, formulas for quality determination of a cut surface depending on the key technological parameters of processing (jet pressure, granularity, the size and abrasive consumption, jet feed, physicomechanical parameters of material, etc.) are lacking. The lack of adequate theoretical models of surface cut roughness does not allow the technologists to carry out optimization of the process taking into account requirements to the set roughness.

\section{Hydroabrasive cutting modeling}

Conducting theoretical researches of hydroabrasive cutting the core regularities of abrasive particles interaction with the surface of the processed workpiece are revealed. Influence of technological parameters of processing on a roughness-cutting surface is investigated as well as the mechanisms of cutting zone formation are identified. While cutting the unevenness of roughness distribution and formation of the so-called zones of smooth and wavy cut are observed. One zone smoothly flows into another. The zone of a smooth cut is a smooth surface with the set roughness, and a zone of a wavy cut is a scalloped surface directing against the cutting jet causing risks. The proportionality of these zones depends on the processing modes.

Based on the dependences for definition of metal removal at a single interaction of abrasive grain when processing a detail with a free abrasive, as offered in works [1,2] and also on results of authors' investigation [3], the dependence for identification of maximum depth of the abrasive cutting particle penetration is received:

$$
h_{\max }=D K_{L} \sin \alpha \sqrt{\frac{2 P_{d y n} \rho_{u}}{3 c \rho_{\text {mix }} k_{s} \sigma_{s}}}
$$

where $h_{\max }$ - density of material particles, $\mathrm{kg} / \mathrm{m}^{3} ; k_{s}$ - coefficient considering influence of detail surface roughness on the true contact area, $P_{d y n}-$ dynamic pressure of mixture, Pa; $\rho_{\text {mix }}-$ density of the water-particles mixture $\mathrm{kg} / \mathrm{m}^{3}, \quad D$ - particle diameter, $\mathrm{m} ; K_{L}-$ coefficient of losses considering distance from a nozzle to a processed detail surface; $c$ coefficient estimating the bearing capacity of a contact area, $\sigma_{s}$ - yield point of the material detail, MPa., $\alpha$ - impact angle of the abrasive particle with the cutting surface, degree.

On the bases of works [1, 2, 3 и 4] the formula of roughness within hydroabrasive cutting is received:

$$
R a=10,18 k_{\alpha}^{R a} \sqrt{\frac{h_{\max } R}{\sqrt{\lambda}}}
$$

где $k_{\alpha}^{R a}$ - coefficient estimating the delivery edge of a jet; $R$-radius of abrasive particles, $\mathrm{m} ; \lambda$ - intensity of a flow of events (number of particles interaction of the abrasive environment with a detail surface per unit time rated per a square unit of packing)

Having substituted the expression of $h_{\max }$ for definition in formula (2) we will receive the dependence for roughness calculation which covers number of interactions in unit of time on the square area of packing $\lambda[4,5]$ : 


$$
R a=10.18 K_{\alpha}^{R a} \sqrt{2 R^{2} \cdot K_{L} \cdot \operatorname{Sin} \alpha \cdot \sqrt{\frac{2 \cdot P_{d y n} \cdot \rho_{u}}{\lambda \cdot 3 \cdot c \cdot \rho_{\text {mix }} \cdot K_{S} \cdot \sigma_{S}}}}
$$

Variable $\lambda$ is a function of feed, quantities of an abrasive in the cutting jet and cut depths. With the help of this variable we can see the influence of particles of the environment. Less nozzle feed of the cutting head, the larger quantity of abrasive particles participates in formation of surface roughness of a cut. Increasing quantity of an abrasive the number of useful impacts with the surface of preformings in the cutting jet grows, so, the roughness of a surface improves. The depth of roughness measurement depends on the amount of grains and energy of interaction with the processed material. Deeper the measurement, smaller energy the particles have, rougher the surface is. $\lambda$ is determined experimentally $[4,5]$.

\section{The results of experimental studies}

For efficient confirmation of the received theoretical dependence the complex of experimental hydroabrasive cutting investigations is carried out.

As an equipment five-access machine for hydroabrasive, cutting "Flow" with a computerized numerical control is used. For sampling, the materials that are often applied to aircraft details fabrication (steel 30HGSA, aluminum D16T alloy, polymer-composite like fibreglass-titanium, etc.) are utilized.

Measurement of roughness was performed on the digital profilometer Surtronic 25 of Taylor Hobson. Statistical data processing was done with the usage of the software of applied and scientific calculations of Mathcad. The study of the influence on surface roughness of a workpiece of such parameters as nozzle feed, discharge rate of an abrasive, physicomechanical material properties, thickness of the processed material were conducted. Data study showed that accelerating nozzle feed leads to the roughness aggravation of a cut surface, the relation of height of a wavy cut zone to a smooth cut zone grows. The experiment was conducted at a graduated increase in feed through each $15 \mathrm{~mm}$ of a cut from $5 \mathrm{~mm} / \mathrm{min}$ up to the size where the through cutting of material was stopped.

On the basis of the obtained data statistical processing and the regression analysis are executed. As a result one and two-factor equations of regression illustrating changes in the surface roughness of a cut with the increase in nozzle feed $S$ and also the depth of roughness measurement (fig. 1) are received. Points on figure 1 and the subsequent figures identify the experimental values, lines and surfaces - regression dependences.

The one-factor equation of regression for finding index value of cut roughness on a depth of measurement of $10 \mathrm{~mm}$ depending on nozzle feed $\mathrm{S}$ looks as follows:

$$
R a_{10}=2.261 \cdot 10^{-6}+7.15 \cdot 10^{-9} \cdot S+5.633 \cdot 10^{-11} \cdot S^{2}
$$

The two-factor equation of regression for finding variable of cutting roughness depending on nozzle feed of $S$ and depth of roughness measurement $h_{\max }$ for the hardened steel 30HGSA looks as following:

$$
\begin{aligned}
R a= & 3.602 \cdot 10^{-6}-3.225 \cdot 10^{-8} \cdot S-1.35 \cdot 10^{-7} \cdot h-1.963 \cdot 10^{-9} \cdot S \cdot h+ \\
+ & 2.439 \cdot 10^{-11} \cdot S^{2} \cdot h+9.569 \cdot 10^{-11} \cdot S \cdot h^{2}+8.116 \cdot 10^{-10} \cdot S^{2}+ \\
& +6.191 \cdot 10^{-11} \cdot h^{2}-5.056 \cdot 10^{-12} \cdot S^{3}-1.202 \cdot 10^{-10} \cdot h^{3}
\end{aligned}
$$




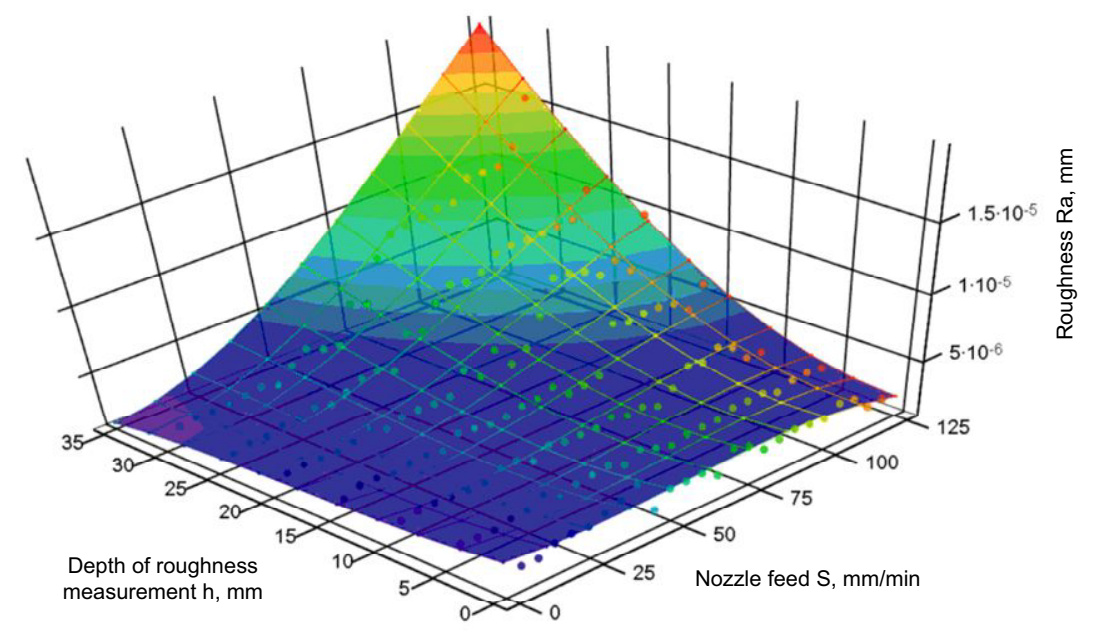

Fig. 1. Dependence of roughness of the steel pattern 30ХГСА with thickness $30 \mathrm{~mm}$ from nozzle feed $\mathrm{S}$ and the depth of roughness measurement.

Experiments 1 studies were also conducted on the hardened steel 30HGSA, aluminum alloy D16T and on polymer - composite materials. It is established that on more solid preformings the surface roughness increases with the supply expansion, the height of a wavy cut zone prevails over a zone of a smooth cut. Through cutting of material is stopped on lower values of supply on the hardened steel in comparison with "crude". Such phenomenon can be described through falling of power jet parameters and effect of "reflection". Colliding with the more solid material of grain the material collapses more intensively and also the large amount of grains jumps aside in the opposite direction, without causing useful interaction, thereby preventing the next grains to get to a cutting zone.

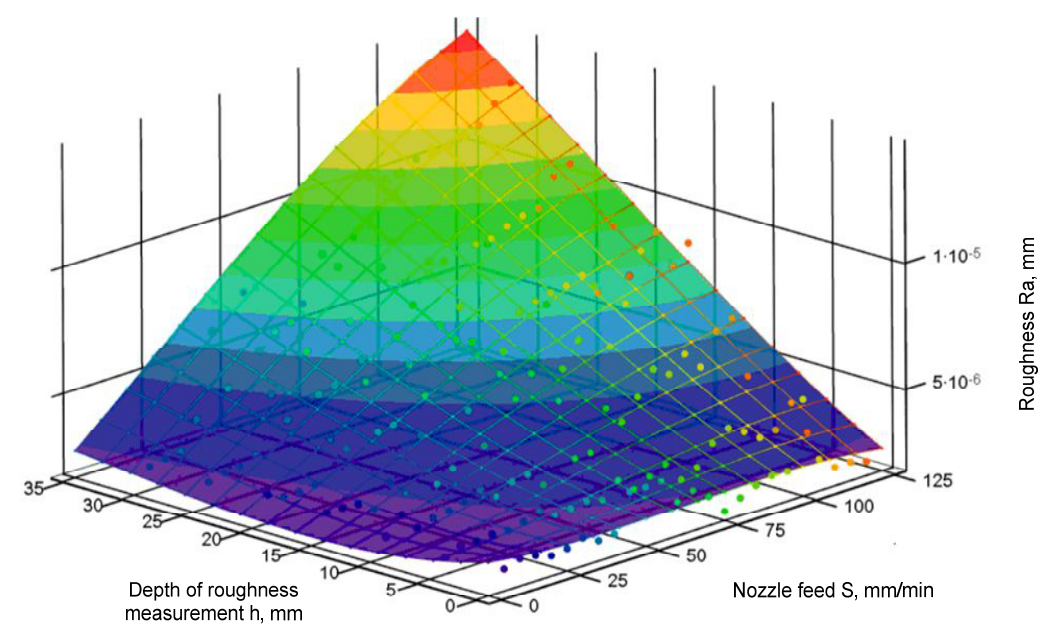

Fig. 2. Dependence of roughness of the steel pattern 30XGSA with thickness $30 \mathrm{~mm}$ from nozzle feed and the depth of roughness measurement.

The two-factor equation of regression for finding the size of roughness of a surface cut depending on feed of $S$ and depth of roughness measurement $h$ for the hardened steel 30HGSA looks as following: 


$$
\begin{gathered}
R a=3.091 \cdot 10^{-6}-1.819 \cdot 10^{-8} \cdot S-1.429 \cdot 10^{-7} \cdot h+4.101 \cdot 10^{-9} \cdot S \cdot h- \\
-6.565 \cdot 10^{-12} \cdot S^{2} \cdot h-1.456 \cdot 10^{-11} \cdot S \cdot h^{2}+3.491 \cdot 10^{-10} \cdot S^{2}+ \\
\quad+6.054 \cdot 10^{-9} \cdot h^{2}-1.852 \cdot 10^{-12} \cdot S^{3}-6.222 \cdot 10^{-11} \cdot h^{3}
\end{gathered}
$$

When carrying out research the mechanism of formation of a wavy cut zone is offered. At feed increase, there is an increase in an angle of particles penetration in detail material due to a larger lag of the cutting jet. At the small angles of penetration, there is a metal removal, formation of the uniform surface. With the angle increase the quantity of the reflected particles grows, the number of the useful interactions with material decreases. The reflected particles interfere with the cutting process and perform a barrier to the particles that are again coming to a zone of cutting. At the same time, there is the continuous motion of a jet in the direction of feed. The angle of penetration grows, and achieving a particular jet deviation, the through penetration of material process happens again. At the same time particles, colliding with each other, start back, and move ahead in the direction of the processed surface, thereby forming deep furrows. At the movement of the cutting jet in the direction of feed the jet deviation, increase in an angle of attack and penetration material repeats again. Thus, the zone of a wavy cut that is similar to waves, consisting of hollows and crests is formed.

The received regression models allow determining roughness of a cut surface in any point by a cut depth and determining smooth and wavy cut.

The number of the useful interactions of grit in unit of time $\lambda$ was defined by comparison of the values received by calculation for the regression models removed according to the experimental data and theoretical dependence for determination of roughness (3). The theoretical value of roughness is paid off at $\lambda=1$.Comparison of theoretical and experimental data indicates that the values calculated by a formula (3) describe rather precisely and adequately the running process. The results' divergence does not exceed $15 \%$.

The transition from a schedule straight line upward illustrates the place of zone change from a smooth cut into a wavy cut causing sharp deterioration in the surface roughness. With the increase of a jet nozzle the size of a wavy cut zone increases, the upturn becomes sharper.

\section{Conclusion}

Based on the conducted research there is an opportunity to execute optimization of hydroabrasive cut by criterion of the least cost of processing. Optimum cutting modes will provide the given roughness at the least prime cost of a cut. The input data will be identified in the form of constant values of processing parameters (initial roughness, jet pressure, cut length, measurement depth, the size of an abrasive particle, etc.) and variable rates of feed and consumption of an abrasive.

Let us carry out calculation of the number of useful impacts $\lambda$. Then we substitute these values to determinate roughness (3). Further, we make comparison of the input and output values of roughness. We eliminate those values that do not meet the requirements of the given roughness. We perform calculation of the prime cut cost, and we sample those values of parameters of processing at which the prime cost of a cut will be minimum.

Application of methodology that optimize technological processes of hydroabrasive cutting passed the production tests in the conditions of the Mechanical plant ROSRVERTOL. 


\section{References}

1. M.A. Tamarkin, Theoretical foundations of the process optimization of detail processing by loose abrasives [The author's thesis] (Rostov-on-Don, 1995)

2. M.A. Tamarkin, Key Engineering Materials, 291, 319 (2005)

3. A. V. Korolev, Ju. K. Novoselov Theoretico-probalistic foundations of abrasive processing (Saratov university, Saratov, 1989)

4. A. V. Verchenko, The efficiency increase of technological processes of hydroabrasive detail cutting [The author's thesis] (Rostov-on-Don, 2017)

5. A. V. Verhenko, Vestnik of DSTU, 2(89),116 (2017) 